\title{
Successful Salvage of a Renal Allograft Where an Infected Lymphocele Caused Pressure-Related Extensive Venous Thrombosis: A Case Report
}

Di Natale, Samuela ; Rössler, Fabian ; Oulare, Fode Bangaly ; de Rougemont, Olivier

\begin{abstract}
Venous Allograft Thrombosis (VAT) is the leading non-immunological cause for early graft loss after kidney transplantation (KT), with a reported prevalence up to $4.2 \%$ [1]. VAT usually has a devastating effect, notoriously difficult to treat and ultimately leading to graft loss in most cases. In contrast, post-transplant lymphocele is a frequent complication after $\mathrm{KT}$, with a reported prevalence up to $33.9 \%$ [2]. However, lymphocele related pressure-induced VAT has not been reported previously. Endovascular Therapy (ET) is to date considered first-line therapy for arterial complications after KT. However, its role for VAT is still unclear and reports are lacking. We present a case of an extended kidney VAT, caused by compression of a large lymphocele and rescued with non-surgical treatment.
\end{abstract}

DOI: https://doi.org/10.46998/ijcmcr.2021.13.000310

Posted at the Zurich Open Repository and Archive, University of Zurich ZORA URL: https://doi.org/10.5167/uzh-215482

Journal Article

Published Version

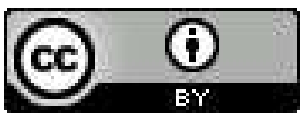

The following work is licensed under a Creative Commons: Attribution 4.0 International (CC BY 4.0) License.

Originally published at:

Di Natale, Samuela; Rössler, Fabian; Oulare, Fode Bangaly; de Rougemont, Olivier (2021). Successful Salvage of a Renal Allograft Where an Infected Lymphocele Caused Pressure-Related Extensive Venous Thrombosis: A Case Report. International journal of clinical studies and medical case reports, 13(2):1-3. DOI: https://doi.org/10.46998/ijcmcr.2021.13.000310 


\title{
Successful Salvage of a Renal Allograft Where an Infected Lymphocele Caused Pressure-Related Extensive Venous Thrombosis: A Case Report
}

\author{
Fabian Rössler, Samuela Di Natale*, Fode Bangaly Oulare, Olivier de Rougemont
}

Department of Surgery and Transplantation, University Hospital Zurich, Zurich, Switzerland

"Corresponding author: Samuela Di Natale, Department of Surgery and Transplantation, University Hospital Zurich, Zurich, Switzerland

Received: August 30, 2021

Published: September 23, 2021

\begin{abstract}
Venous Allograft Thrombosis (VAT) is the leading non-immunological cause for early graft loss after kidney transplantation (KT), with a reported prevalence up to $4.2 \%$ [1]. VAT usually has a devastating effect, notoriously difficult to treat and ultimately leading to graft loss in most cases. In contrast, post-transplant lymphocele is a frequent complication after KT, with a reported prevalence up to $33.9 \%$ [2]. However, lymphocele related pressure-induced VAT has not been reported previously. Endovascular Therapy (ET) is to date considered first-line therapy for arterial complications after KT. However, its role for VAT is still unclear and reports are lacking.
\end{abstract}

We present a case of an extended kidney VAT, caused by compression of a large lymphocele and rescued with non-surgical treatment.

\section{Case Report}

A 63-year-old patient underwent KT for diabetic nephropathy. A left kidney from a 55-year-old male donor after circulatory death was transplanted to the right external iliac vessels. One vein and three arteries on one single patch, were anastomosed. Intra- and postoperative course was surgically uneventful with an operation time of 142 minutes, anastomotic time (both vein and arteries) of 36 minutes and cold ischemic time of 418 minutes. The patient developed Delayed Graft Function (DGF) and needed dialysis for thirteen days after transplantation. Repeated ultrasound confirmed normal perfusion and flow rates of the graft (Figure 1). The patient was discharged the 9th day, without any surgical complications and steadily improving graft function.

On day 15 th secretion from the wound was detected. Computed tomography revealed an encapsulated fluid collection $(6 \times 3 \mathrm{~cm})$ around the graft (Figure 2). Creatinine values in the fluid were normal, so suspicion of a lymphocele was raised. Due to the steadily improving graft function and without signs for allograft or ureteric compression, we decided to proceed conservatively. Kidney function at 5 weeks was good with a creatinine of $144 \mathrm{umol} / \mathrm{l}$, an estimated glomerular filtration rate of $44 \mathrm{ml} / \mathrm{min}$.

Starting from week 6 after transplantation, episodes of intermittent severe proteinuria appeared. Initially, ultrasounds confirmed normal perfusion without any signs of VAT- Biopsy revealed severe tubular damage and regeneration. Kidney function steadily decreased. An ultrasound follow-up revealed a partial VAT (Figure 3) with a thrombus from the lower leg to the graft vein. Immediately, therapeutic anticoagulation with heparin was started. Because of the suspicion of a pressure induced VAT, the lymphocele was drained percutaneously. Microbiological samples revealed enterobacter cloacae, antibiotic therapy was initiated.

Despite four days of anticoagulation, thrombosis was persistent and graft function deteriorated further, up to a peak serum creatinine of $854 \mathrm{umol} / 1$. The patient had preserved diuresis and no need for dialysis. That's why we decided against a reoperation and proceeded with percutaneous placement of a lysis catheter via the femoral vein for 24 hours (Actilyse(C). The day after severe stenosis of the venous anastomosis and common iliac vein were treated by Percutaneous Transluminal Angioplasty (PTA). However, no complete recanalization could be

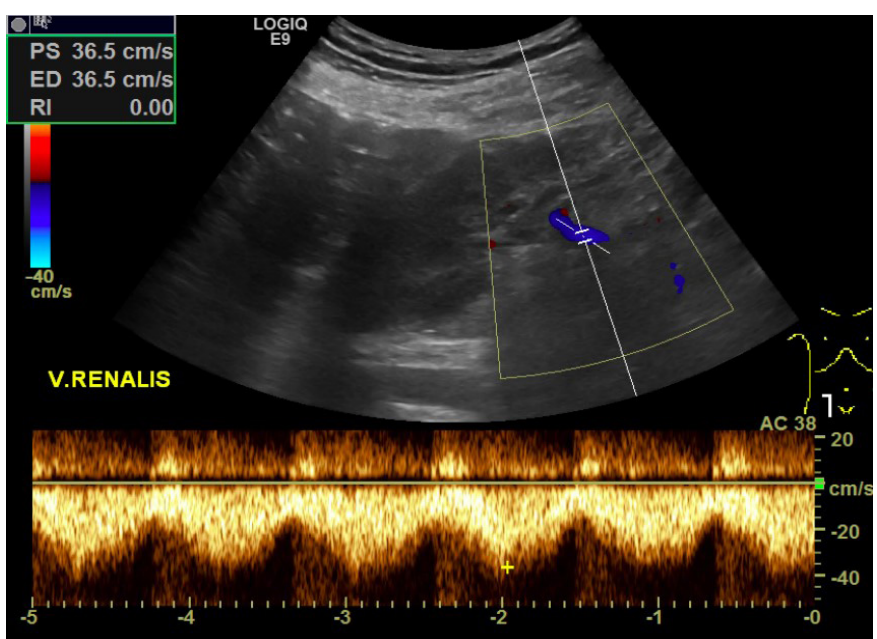

Figure 1: Duplex ultrasonography on the 4th postoperative day shows a regular venous signal. 


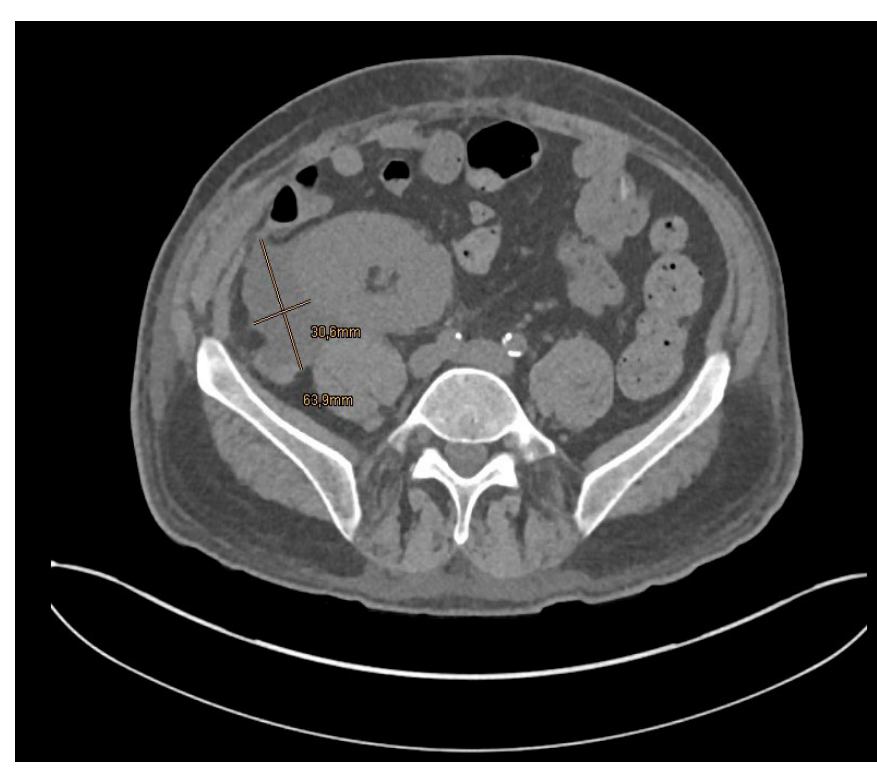

Figure 2: Encapsulated fluid collection around the allograft on the 15th postoperative day.

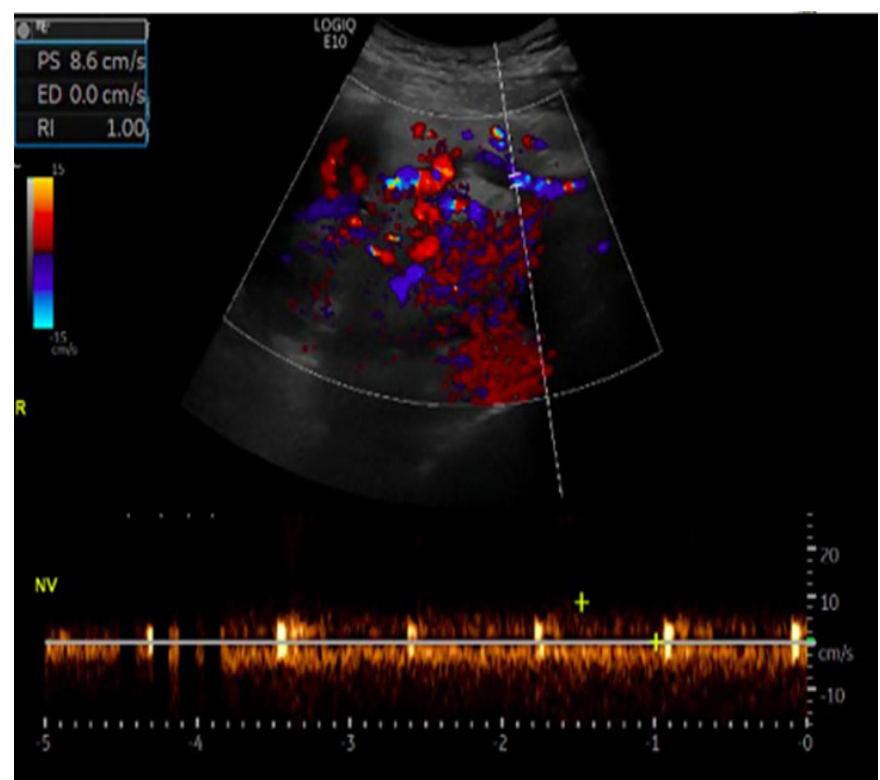

Figure 3: Duplex ultrasonography performed two months postoperatively shows a partial VAT.

achieved (Figure 4). Under continuous anticoagulation serum creatinine levels dropped constantly.

At last follow-up, one year after transplantation and 10 months after thrombosis, graft function was stable with a serum creatinine of 139 umol/1, equal to a glomerular filtration rate of $46 \mathrm{ml} / \mathrm{min}$ according to the Chronic Kidney Disease Epidemiology Collaboration equation. The patient is kept under oral anticoagulation (Marcoumar(C).

\section{Discussion}

Pressure-related complications after KT are rare and to our knowledge, there are no reports on VAT caused by compression from a lymphocele. Management of VAT after KT is difficult and often frustrating. Reoperation has generally poor success rates and often results in graft loss [3].

The role of ET in VAT remains unclear. Even though, ET has been described as safe for iliac vein thrombosis4, only few small series report successful graft salvage with ET [4]. In this case, its benefit was as well questionable. The combination of local lysis and PTA did not decisively improve recanalization. Heparinization and lymphocele drainage probably provided

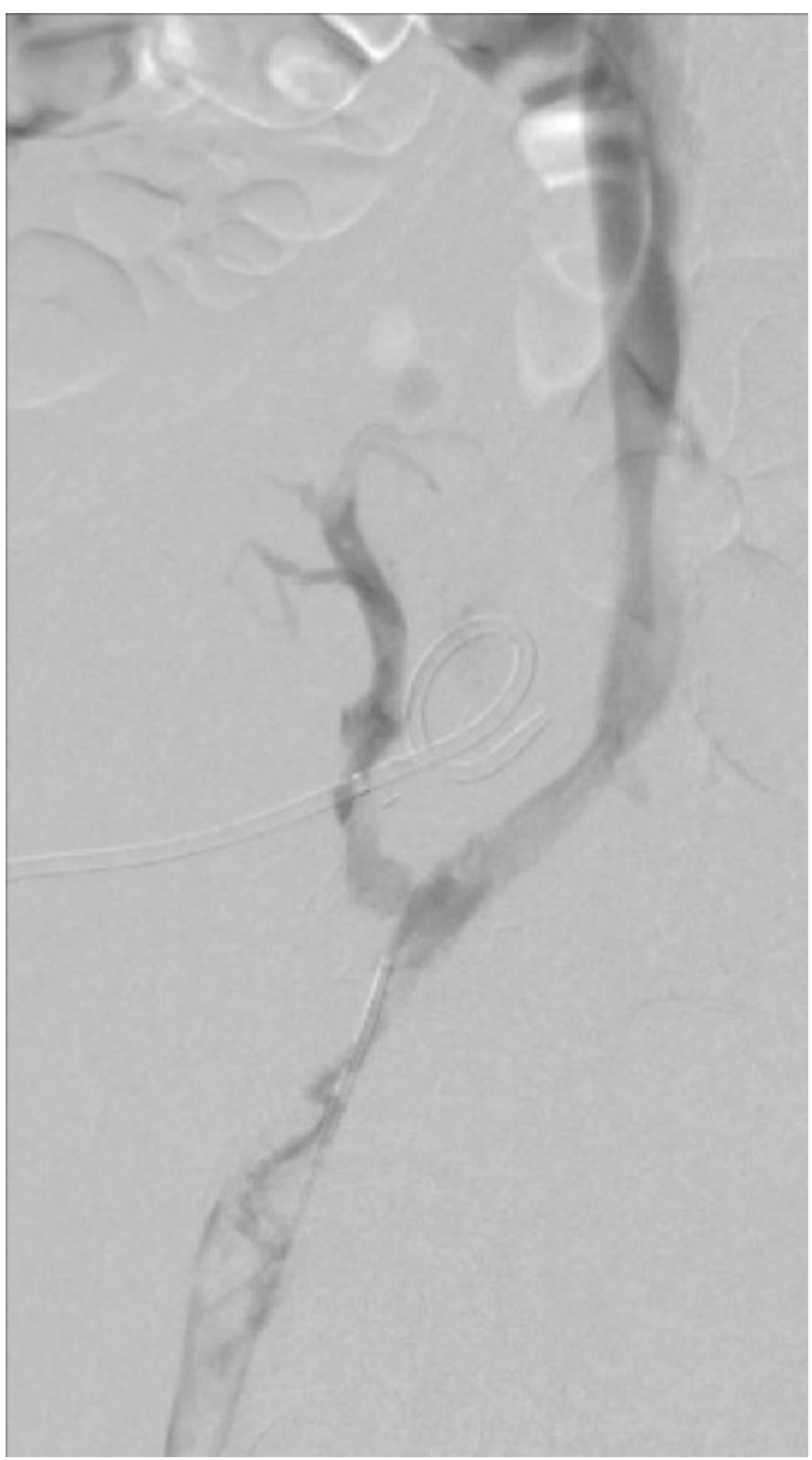

Figure 4: Angiography shows incomplete recanalization of the renal and iliac vein after angioplasty and lysis. The percutaneous drainage of the infected lymphocele is still in situ.

the greatest benefit for graft salvage.

Importantly, urinary output always remained preserved and there was never an indication for dialysis. This, combined with a high risk for graft loss in reoperation, convinced us not to reoperate and continue with anticoagulation and fluid drainage only, even after the questionable success of ET.

The most important risk factors for VAT are technical issues and hypercoagulative staten [5]. However, in our patient, surgery was uneventful with standard end-to-side running vascular anastomosis, short operative and cold ischemic time. Second, thrombosis occurred later than usual, almost six weeks after transplantation. This is unusual, as thrombosis related to technical problems occurs usually early, within 2 weeks after KT [3]. In this case venous thrombosis was not complete and perfusion of the graft was present at all times. It was most likely caused by external compression of an infected lymphocele. This case demonstrates successful non-surgical treatment of a kidney VAT through a combination of therapeutic anticoagulation and lymphocele decompression, restoring graft function with sustained success at 1-year follow-up. Here, the benefit of ET remains unclear. 


\section{References}

1. El Zorkany K, Bridson JM, Sharma A, Halawa A. Transplant Renal Vein Thrombosis. Experimental and clinical transplantation : official journal of the Middle East Society for Organ Transplantation. 2017; 15(2): 123-129.

2. Ranghino A, Segoloni GP, Lasaponara F, Biancone L. Lymphatic disorders after renal transplantation: new insights for an old complication. Clinical kidney journal. 2015; 8(5): 615-622.

3. Aschwanden M, Thalhammer C, Schaub S, Wolff T, Steiger J, Jaeger KA. Renal vein thrombosis after renal transplantation--early diagnosis by duplex sonography prevented fatal out- come. Nephrology, dialysis, transplantation : official publication of the European Dialysis and Transplant Association - European Renal Association. 2006; 21(3): 825-826.

4. Xue GH, Huang XZ, Ye M, et al. Catheter-directed thrombolysis and stenting in the treatment of iliac vein compression syndrome with acute iliofemoral deep vein thrombosis: outcome and follow-up. Annals of vascular surgery. 2014; 28(4): 957-963.

5. Dimitroulis D, Bokos J, Zavos G, et al. Vascular complications in renal transplantation: a single-center experience in 1367 renal transplantations and review of the literature. Transplantation proceedings. 2009; 41(5): 1609-1614.16 